\title{
Alterstice
}

Revue internationale de la recherche interculturelle

International Journal of Intercultural Research

Revista International de la Investigacion Intercultural

\section{Development of Geocentric Spatial Language and Cognition: An Eco-Cultural Perspective, de P. Dasen et R. Mishra}

\section{Camille Brisset}

Volume 1, numéro 1, 2011

URI : https://id.erudit.org/iderudit/1077594ar

DOI : https://doi.org/10.7202/1077594ar

Aller au sommaire du numéro

Éditeur(s)

Alterstice

ISSN

1923-919X (numérique)

Découvrir la revue

Citer ce compte rendu

Brisset, C. (2011). Compte rendu de [Development of Geocentric Spatial

Language and Cognition: An Eco-Cultural Perspective, de P. Dasen et R. Mishra].

Alterstice, 1(1), 95-99. https://doi.org/10.7202/1077594ar d'utilisation que vous pouvez consulter en ligne.

https://apropos.erudit.org/fr/usagers/politique-dutilisation/ 


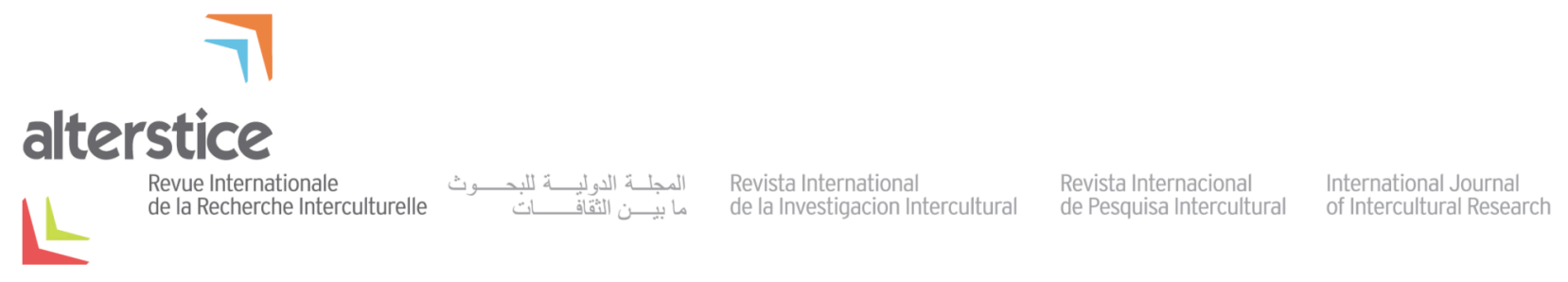

NOTE DE LECTURE

\title{
Development of Geocentric Spatial Language and Cognition: An Eco-Cultural Perspective, de P. Dasen et R. Mishra
}

Camille Brisset ${ }^{1}$

\author{
Rattachement de l'auteure \\ ${ }^{1}$ Laboratoire Psychologie et Cultures, École de Psychologie, Université Laval, Québec, Canada

\section{Correspondance} \\ camille.brisset.1@ulaval.ca
}

\section{Références de l'ouvrage :}

Dasen, P. et Mishra, R. (2010). Development of Geocentric Spatial Language and Cognition: An Eco-Cultural Perspective. Cambridge : Cambridge University Press. (ISBN : 978-0521191050)

\section{Pour citer cet article :}

Brisset, C. (2011). Development of Geocentric Spatial Language and Cognition: An Eco-Cultural Perspective [Note de lecture]. Alterstice, 1(1), 95-100.

Cet ouvrage de 408 pages rédigé en anglais s'inscrit dans les domaines de la psychologie du développement cognitif et de la psychologie interculturelle. Les auteurs, Pierre R. Dasen et Ramesh C. Mishra, s'intéressent au développement des enfants en interaction avec la culture, et plus précisément au développement du langage et de la cognition spatiale géocentrique en Indonésie, en Inde, au Népal et en Suisse. "Mets ton couteau au nord de ton assiette, et ta fourchette au sud ${ }^{1}$ ! " illustre très bien la réalité explorée dans le livre. Aussi surprenante que soit cette formulation, elle renvoie à un langage, peu utilisé dans les pays occidentaux, dont le cadre de référence (CdR) est dit géocentrique. À l'inverse, dans un CdR égocentrique, un individu parlera davantage de la gauche ou de la droite de l'assiette, du haut ou du bas.

Pierre R. Dasen est professeur honoraire émérite en approches interculturelles de l'éducation à la Faculté de Psychologie et des Sciences de l'Éducation de I'Université de Genève (Suisse). S'inscrivant dans le champ de la psychologie développementale interculturelle, il s'est questionné tout au long de sa carrière sur l'interaction entre culture et cognition. Il est aussi un des fondateurs de l'Association pour la Recherche Interculturelle (ARIC, en 1984) et membre honoraire de I'International Association for Cross-Cultural Psychology (IACCP). Ceux qui connaissent Pierre R. Dasen vous diront que cet ouvrage reflète une grande partie de son parcours personnel.

\footnotetext{
1 "Put the knife on the north side of the plate, and the fork south! " (Dasen et Mishra, 2010, p. 3).
} 
Ramesh C. Mishra, quant à lui, est professeur au Département de Psychologie, Faculté des Sciences Sociales de Banaras Hindu University (Varanasi, Inde). Ses travaux s'ancrent dans le champ de la psychologie interculturelle et il s'intéresse principalement aux influences culturelles sur le développement humain. Il a également contribué à de nombreuses publications scientifiques en Inde et à l'étranger, et ce, dans les domaines de la cognition, de l'acculturation, de la scolarisation et des études interculturelles.

Ce livre est le résultat d'une vingtaine d'années de recherche et d'une décennie de collaboration entre les deux auteurs qui, au travers de 15 chapitres répartis en quatre parties, nous montrent comment les enfants de 4 à 15 ans se construisent un $\mathrm{CdR}$ géocentrique dans le langage tant verbal que non-verbal.

\section{Partie 1 - Introduction et méthode}

Le premier chapitre présente les théories sous-jacentes aux questionnements des auteurs, soulignant ainsi les débats sur l'universalité et la relativité du développement humain. Dasen et Mishra optent à cet égard pour une position intermédiaire : ils pensent que les processus cognitifs de base sont universels, mais qu'ils s'expriment différemment selon les cultures. Ainsi, les différences culturelles observées dans la cognition découleraient davantage des styles cognitifs prédominants dans chaque culture, du fait des demandes de l'environnement, que de la présence ou de l'absence de processus cognitifs propres à une culture donnée. Cette argumentation est également valable pour la cognition spatiale et pour le " choix » entre CdR égocentrique et CdR géocentrique. Les travaux de Piaget et Inhelder (1948/1956) et les recherches en linguistique comparée de Levinson (1996, 2003) sur le développement des CdR spatiaux sont les points de départ de cet ouvrage; le modèle éco-culturel intégratif de Dasen (2003) donne aux auteurs un cadre théorique pertinent pour en étudier le développement dans une perspective interculturelle. Dasen et Mishra s'inscrivent également, mais modérément, dans le paradigme de Levinson (2003) sur le relativisme linguistique. Ce dernier postule que "la langue détermine l'utilisation d'un cadre égocentrique ou bien géocentrique du fait de processus cognitifs non linguistiques, comme l'encodage d'un dispositif spatial en mémoire » (Dasen, Mishra, Niraula et Wassmann, 2006, p. 147).

Partant du fait qu'il est nécessaire - voire indispensable - de comprendre la culture dans laquelle se déroule la recherche (le choix entre CdR égocentrique et CdR géocentrique reposant en grande partie sur l'environnement écologique), mais aussi d'adapter "culturellement » les outils aux populations étudiées, tout en maintenant leur validité, les chapitres 2 et 3 se veulent méthodologiques. Le premier décrit les épreuves verbales et non-verbales utilisées, adaptées de Levinson (2003), et le second les contextes culturels (dont les systèmes d'orientation spatiale) dans lesquels ces dernières ont été administrées.

\section{Partie 2 - Résultats}

Le chapitre 4 présente les résultats de l'étude dite "pilote " conduite en 1994 à Bali (Indonésie), de même que ceux des recherches initiales menées en Inde (Roopchandpour et Varanasi) et au Népal (dans la région de Dolakha) en 1999-2000. C'est notamment à Bali que les auteurs ont découvert, chez l'enfant, l'existence du CdR géocentrique tant à un niveau verbal que non-verbal, et ce dès l'âge de 4 ans. Ils concluent que les deux $C d R$ coexistent dans chaque individu, quelle que soit la culture, soulignant ainsi l'universalité des processus cognitifs, et que ce n'est pas tant la langue qui détermine leur recours, mais plutôt la situation. Des influences écologiques et acculturatives y sont également soulignées, en faveur de la relativité du développement humain. Ces trois premières études ont soulevé un certain nombre de questionnements qui ont conduit les auteurs à l'étude principale de cet ouvrage, menée dans cinq lieux différents entre 2002 et 2007.

Le chapitre 5 en expose les résultats, obtenus à Bali en 2002. Dasen et Mishra corroborent entre autres leurs conclusions de 1994 concernant l'acquisition précoce du langage géocentrique, mais seulement en milieu rural et chez les enfants ne parlant que le balinais. Pour rendre compte des différences observées entre ces derniers et ceux parlant l'indonésien, ils soulignent l'impact du processus d'acculturation chez les enfants parlant l'indonésien. De plus, les résultats indiquent que la langue ne serait pas le seul et unique déterminant de la cognition. Les CdR apparaissent profondément liés au contexte éco-culturel et la langue n’en est pas le seul élément constitutif. La pertinence du système d'orientation de la topographie locale, ou encore les aspects symboliques et religieux, en sont d'autres composantes.

Alterstice - Revue Internationale de la Recherche Interculturelle, vol. $1, n^{\circ} 1$ 
Le chapitre 6 présente les résultats de l'étude principale conduite à Varanasi (Inde). Les auteurs mettent notamment en avant l'influence du lieu de scolarisation sur l'usage du CdR géocentrique, et ce, en faveur des écoles sanskrit, écoles "traditionnelles » indiennes (à l’opposé des écoles hindi, plus occidentalisées). En accord avec les conclusions de l'étude de Bali, la variabilité observée dans les processus cognitifs spatiaux ne peut être réduite à l'unique influence de la langue. Il existe un ensemble de variables qui constitue l'environnement écologique dont le rôle varie dans l'organisation du langage et de la cognition spatiale; toutefois, les aspects socioculturels ressortent de façon moins marquée que dans les résultats de l'étude menée à Bali. Les auteurs rapportent par exemple un lien en faveur du CdR égocentrique avec l'accès aux médias (en tant que mesure indirecte du processus d'acculturation) ou encore, l'influence des connaissances sur le système cardinal, d'une part, et des habiletés spatiales sur le langage géocentrique, d'autre part.

Ces premiers résultats s'inscrivent clairement à mi-chemin dans le débat entre l'universalité et la relativité du développement humain. La coexistence des deux $C d R$ tant à Bali qu'à Varanasi est un élément en faveur de l'universalité, les variabilités écologiques et culturelles observées dans l'expression de ces CdR, en faveur de la relativité. Se pose maintenant la question de savoir ce qu'il en est « ailleurs ».

Le chapitre 7 est centré sur les résultats obtenus à Katmandou, au Népal. Cette ville a été choisie comme lieu de récolte de données suite aux différences observées selon la langue parlée à Bali (le balinais étant considéré comme ayant un CdR géocentrique et l'indonésien, un CdR égocentrique). En effet, à Katmandou, les auteurs avaient la possibilité d'étudier le langage et la cognition spatiale chez des enfants bilingues. Considérant que les deux CdR coexistent chez un même individu et que le népalais devrait favoriser un CdR géocentrique et l'anglais, un CdR égocentrique, Dasen et Mishra ont comparé des enfants scolarisés dans des écoles anglaises et népalaises pour constater avec surprise qu'apprendre en anglais ne suscite pas de préférence particulière pour le CdR égocentrique tant au niveau verbal que non-verbal. Ce qui importerait, c'est le langage normalement parlé dans la communauté. De plus, en croisant les données de cette étude avec celle de 1999-2000 (Dolakha), les auteurs corroborent leurs conclusions sur le contraste écologique entre ville et campagne : il y a une prédominance du langage géocentrique dans les deux localisations, mais absence du langage égocentrique en milieu rural (Dolakha) alors qu'il apparaît en milieu urbain (Katmandou).

Le chapitre 8 présente les résultats obtenus à Panditpour, village de campagne en Inde. Les auteurs ont convenu de ce lieu pour mettre à l'épreuve l'hypothèse écologique décrite ci-dessus. En effet, ses habitants y utilisent un langage égocentrique pour donner des directions. Cependant, les résultats aux épreuves piagétiennes vont dans le sens d'une préférence pour le CdR géocentrique, tant chez les adultes que chez les enfants. Autant les auteurs ne semblent pouvoir expliquer pourquoi les habitants de Panditpour n'ont pas eu recours au langage égocentrique, autant le CdR géocentrique apparaît clairement comme dominant dans le langage en milieu rural (ce qui va dans le sens de l’hypothèse écologique).

Le chapitre 9 concerne les résultats obtenus à Genève. Cette ville suisse a été choisie du fait de la nécessité d'un groupe contrôle. Même si Dasen savait implicitement que les enfants occidentaux n'utilisent pas de CdR géocentrique, il était nécessaire de le vérifier empiriquement afin de permettre une comparaison. Les données obtenues vont dans ce sens: les enfants parlant français n'ont pour ainsi dire jamais recours au langage géocentrique. Toutefois, le fait que deux des plus âgés aient donné de telles réponses questionne les auteurs sur la possibilité d'une flexibilité dans les CdR (égocentrique ou géocentrique) selon la tâche.

Ainsi, il ressort des différents chapitres que tous les enfants, qu'ils soient suisses, népalais, balinais ou indiens, peuvent élaborer différents $C d R$, et que l'expression que vont prendre ces derniers dépend clairement des demandes de l'environnement.

\section{Partie 3 - Recherches complémentaires}

Dans la troisième partie de cet ouvrage, Dasen et Mishra présentent toute une série d'études additionnelles, non pas parce que les questions auxquelles ils tentent de répondre sont moins importantes, mais parce qu'elles sont spécifiques à certaines localisations ou certains échantillons. 
Le chapitre 10 s'intéresse au développement du langage spatial chez des enfants qui ont grandi dans un contexte où le langage géocentrique et l'utilisation d'un système d'orientation géocentrique sont la norme chez l'adulte. À travers trois études, deux à Roopchandpour (Inde) et une à Dolakha (Népal), les auteurs nous montrent que le langage spatial, tout comme n'importe quelle composante du comportement humain, s'adapte aux conditions écologiques et culturelles locales. Les enfants apprennent bien de leurs parents le langage spatial, mais au fur et à mesure qu'ils évoluent dans leur environnement, ils acquièrent de nouvelles manières de gérer les réalités spatiales, s'adaptant ainsi au mieux à leur environnement.

L'existence d'un langage déictique, langage "intermédiaire » entre égocentrique et géocentrique ("Là-bas/parlà » accompagnées par un mouvement du doigt) ont amené les auteurs à se questionner, plus spécifiquement dans le chapitre 11, sur la précocité du CdR géocentrique à travers la gestuelle, et donc avant l'acquisition du langage (géocentrique). II s'avère que chez les enfants népalais à Katmandou où les deux CdR sont utilisés au quotidien, le $\mathrm{CdR}$ géocentrique dans la gestuelle prédomine également sur le $\mathrm{CdR}$ égocentrique. La trajectoire développementale observée ici apparait inverse à celle des enfants occidentaux, et la question qui se pose maintenant est de savoir à quoi est due cette inversion.

Dans le chapitre 12, Dasen et Mishra regardent de plus près comment les enfants qui utilisent un CdR géocentrique organisent et décrivent un dispositif spatial. Ils cherchent notamment à savoir si ce dernier sera décrit de la même manière en variant les points de vue (après déplacement des enfants autour du dispositif ou en faisant la rotation du dispositif). Quel que soit le CdR dominant (à Bali, Varanasi, Katmandou, Panditpour ou Genève), la plupart des enfants décrivent la situation de façon différente selon la variation apportée; la tendance à décrire de manière identique ou pas est, selon les auteurs, intimement liée aux schèmes utilisés pour organiser le dispositif spatial en question.

Les recherches en neurologie portant principalement sur le $C d R$ égocentrique, les auteurs étudient de plus près, dans le chapitre 13, les corrélats neurophysiologiques de l'espace géocentrique. D'une part, dans des études conduites à Varanasi et Katmandou, le recours au CdR géocentrique n'apparait pas en lien avec la latéralisation du cerveau, mesurée à un niveau périphérique, alors le $\mathrm{CdR}$ égocentrique serait davantage lié à l'hémisphère droit (voir Galati, Lobel, Vallar, Berthoz, Pizzamiglio et Le Bihan, 2000). D’autre part, les résultats d'une recherche complémentaire à Varanasi auprès de patients ayant des lésions au cerveau suggèrent notamment que ces atteintes n'influenceraient pas l'utilisation de ce $C d R$, alors que de telles lésions semblent affecter le recours à un CdR égocentrique (voir Beschin, Cocchini, Della-Sala et Logie, 1997). Les circuits neuronaux impliqués dans les CdR ne semblent donc pas être les mêmes. Ces résultats sont toutefois exploratoires et restent à approfondir. Les conclusions qui en découlent doivent donc être considérées en conséquence.

Le chapitre 14 s'intéresse au concept de "dead reckoning " ou de navigation à l'estime. II s'agit d'un processus au travers duquel un individu estime continuellement sa position dans l'environnement. Les explorateurs tels que Christophe Colomb qui traversaient l'Atlantique «à l'aveugle » y avait recours: ils évaluaient la vitesse de déplacement ainsi que l'influence de l'environnement (courant, vent) afin de deviner leur position. Le processus apparait clairement comme égocentrique dans la majorité des écrits. Cependant, ces recherches portent uniquement sur des populations occidentales et la question que se posent les auteurs dans ce chapitre est de savoir comment les enfants dont le CdR est principalement géocentrique " emportent " avec eux leurs repères spatiaux dans différents lieux. Cette navigation à l'estime géocentrique a été étudiée dans les écoles sanskrite et hindi de Varanasi (Inde). Les enfants s'avèrent être de bons " navigateurs ", mais parler un langage géocentrique n'apparait pas comme une condition suffisante pour une bonne navigation.

\section{Partie 4 - Conclusions}

Que conclure de tous ces résultats? Et comment les interpréter à la lumière des débats sur l'universalité et la relativité du développement humain, du paradigme sur le relativisme linguistique (Levinson, 2003) ou encore du modèle éco-culturel intégratif (Dasen, 2003)? Le $15^{\mathrm{e}}$ et dernier chapitre répond très clairement à ces questions. Les auteurs expliquent notamment pourquoi ils considèrent les CdR géocentrique/égocentrique comme des styles cognitifs, pourquoi le processus de base sous-jacent au langage et à la cognition spatiale est universel, en quoi 
I'hypothèse de Levinson (2003) est valable au niveau groupal, mais dans une moindre mesure au niveau individuel, et en quoi l'écologie, les systèmes culturels de croyances et l'acculturation jouent un rôle particulier dans le recours à un $\mathrm{CdR}$ spécifique.

\section{Commentaires}

La lecture de ce livre a été un voyage à la fois touristique et scientifique de 15 chapitres. Touristique, parce qu'on y découvre des systèmes d'orientation spatiale différents, des cultures qui nous sont peu familières. Scientifique, parce que ce livre est d'une grande richesse, tant par le nombre et la qualité des références bibliographiques (près de 400), que par le souci permanent des auteurs à considérer les travaux touchant de près ou de loin au domaine du développement du langage et de la cognition spatiale, ou encore, par les apports des résultats. II a également été très appréciable de savoir, dès les premières lignes, à quoi Dasen et Mishra faisaient référence. "Mets ton couteau au nord de ton assiette, et ta fourchette au sud! " est une expression tellement peu familière qu'elle attire tout de suite l'attention et suscite la curiosité du lecteur.

En écrire une note de lecture a toutefois été un exercice difficile, tant cet ouvrage est à la fois complexe et précis. Une lecture attentive et plusieurs "retours en arrière » pour en saisir les (nombreux) détails auront été nécessaires. Autant une maîtrise absolue de la théorie piagétienne n'est pas essentielle, autant des connaissances dans le domaine ne sont pas superflues.

L'œuvre de Dasen est reconnue dans le domaine de la psychologie interculturelle, mais les travaux des deux chercheurs de cet ouvrage méritent d'être davantage connus par la communauté universitaire. Leurs apports donnent, à notre avis, des éléments de réflexion à la fois néo-piagétiens et aussi " néo-occidentaux » intéressants à exploiter.

\section{Références bibliographiques}

Beschin, N., Cocchini, G., Della Sala, S. et Logie, R. (1997). What the eyes perceive, the brain ignores: A case of pure unilateral representational neglect. Cortex, 33, 3-26.

Dasen, P. et Mishra, R. (2010). Development of Geocentric Spatial Language and Cognition: An Eco-Cultural Perspective. New York : Cambridge University Press.

Dasen, P. (2003). Theoretical frameworks in cross-cultural developmental psychology: An attempt at integration. Dans T. S. Saraswathi (dir.), Cross-cultural perspectives in human development: Theory, research, \& applications (p. 128-165). New Delhi : Sage India.

Dasen, P., Mishra, R., Niraula, S. et Wassmann, J. (2006). Développement du langage et de la cognition spatiale géocentrique. Enfance, 2(58), 146-158.

Galati, G., Lobel, E., Vallar, G., Berthoz, A., Pizzamiglio, L. et Le Bihan, D. (2000). The neural basis of egocentric and allocentric coding of space in humans: a functional magnetic resonance study. Experimental Brain Research, $133,156-164$.

Levinson, S. (1996). Frames of reference and Molyneux's question: Cross- linguistic evidence. Dans P. Bloom, M. Peterson, L. Nadel et M. Garrett (dir.), Language and space (p. 109-169). Cambridge, MA : MIT Press.

Levinson, S. (2003). Space in Language and Cognition: Explorations in Cognitive Diversity. New York : Cambridge University Press.

Piaget, J. et Inhelder, B. (1948/1956). The child's conception of space. New York : Norton. 